\title{
Theory of Crack Spacing in Concrete Pavements
}

\author{
By Ann Ping Hong, ${ }^{1}$ Yuan Neng $\mathrm{Li}^{2}$ and Zdeněk P. Bažant, ${ }^{3}$ Fellow, ASCE
}

\begin{abstract}
Agstract: A simple analytical model is developed to predict the average crack spacing and crack depth in highway pavements due to thermal loading. The pavement is modeled as a beam on a Winkler elastic foundation. The effect of cracks on the pavement is considered on the basis of compliance functions. A simple method is introduced to describe the behavior of the pavement material according to nonlinear fracture mechanics. It is shown that the material length in the fracture model should be defined by the total fracture energy, rather than the effective fracture energy. The effect of nonlinearity in the distribution of thermal stress across the pavement depth is also analyzed. The foundation of the pavement is found to have little importance. The theoretical predictions are shown to compare well with field observations on asphalt pavements.
\end{abstract}

\section{INTRODUCTION}

The service life of asphalt concrete pavements as well as portland cement concrete pavements is often determined by cracking. Cracking is caused mainly by temperature and humidity changes. The extent of cracking is usually measured by the cracking index, which represents the total length of visible cracks per unit surface area. The most important for durability, however, is the onset of cracking, which is the focus of this study. In portland cement concrete pavements, joints are placed at a certain spacing to prevent cracking. If the joints are too close, the cost of construction increases and the ride quality decreases. But if the joints are too sparse, cracks are not prevented.

A physically justified theory governing the initiation and spacing of cracks in pavements needs to be formulated to answer these important questions. This is the objective of the present study. Whether surface cracks can start to develop is indicated by the strength criterion. However, the question of whether cracks of finite length can form and what their spacing will be, can be decided neither by the strength criterion, nor the energy criterion of classical fracture mechanics, because the energy release rate vanishes when the crack length is zero.

In studying the hot-dry-rock geothermal energy scheme, Bažant and Ohtsubo (1977) [and in more detail Bažant et al. (1979)] proposed that cracks of a certain finite length form suddenly (or simultaneously over their entire length). In that case the total energy release is finite. By equating its approximate estimate to the energy required to create the cracks, these investigators formulated a condition from which they could obtain realistic predictions of the spacing of thermal cracks in granite [see also Bažant and Cedolin (1991), Eq. 12.6.3]. However, they used an empirical estimate of the fraction of energy release that goes into cracking. This may be avoided by introducing two additional conditions that must be satisfied simultaneously: the stress before cracking must overcome the tensile strength of the material, and the cracks of interest are only those that can propagate further. The second condition means that the initial, suddenly formed cracks of finite length must be critical, i.e., their energy release rate must be equal to the

\footnotetext{
'Predoctoral Res. Assoc., Dept. of Civ. Engrg., Northwestern Univ., Evanston, IL 60208; formerly, Grad. Student, Dept. of Civ. Engrg., The Univ. of Akron, Akron, OH 44325.

${ }^{2}$ Res. Sci. in the rank of Asst. Prof., Dept. of Civ, Engrg., Northwestern Univ., Evanston, IL.

${ }^{3}$ Walter P. Murphy Prof. of Civ. Engrg. and Mat. Sci., Dept. of Civ. Engrg., Northwestern Univ., Evanston, IL; e-mail: z-bazant@nwu.edu.

Note. Associate Editor: Robert Y. Liang. Discussion open until August 1, 1997. To extend the closing date one month, a written request must be filed with the ASCE Manager of Journals. The manuscript for this paper was submitted for review and possible publication on March 20, 1995. This paper is part of the Journal of Engineering Mechanics, Vol. 123. No. 3, March, 1997. CASCE, ISSN 0733-9399/97/0003-0267$0275 / \$ 4.00+\$ .50$ per page. Paper No. 10330 .
}

fracture energy of the material. These two additional conditions were introduced in Li and Bažant's (1994) study of the initial spacing of radial bending through cracks in sea ice emanating from a penetrating object and in the subsequent $\mathrm{Li}$ et al. (1995) study of parallel thermal cracks in a half-space. The purpose of this study is to apply these conditions to a pavement plate supported on an elastic foundation, taking at the same time into account that the material is not perfectly brittle but quasibrittle.

The crack formation and spacing in pavements is also subjected to strong random influences, such as random microstructure or random variability of material strength and toughness. However, certain deterministic conditions must be followed in the average sense. For instance, the law of energy conservation, which is the cornerstone of fracture mechanics, must not be violated during the crack formation. This study will be confined to a deterministic treatment, which approximately describes the mean behavior. One must nevertheless be aware of the random scatter that is superposed on the mean predictions.

As another simplification, we will ignore the rate dependence of crack growth in concrete as well as the viscoelasticity of the material. This has been, of course, a standard feature of fracture analysis of concrete structures, known to be acceptable for short-time loads whose durations fall within one order of magnitude only. Such a simplification is also acceptable for long-time loading (such as thermal stresses of longer duration), provided that the value of fracture energy appropriate for the given load duration is considered and that creep is approximately taken into account by means of the effective elastic modulus.

\section{MECHANICAL MODELING OF PAVEMENT STRUCTURE}

The pavement is structurally modeled as an elastic plate supported on a Winkler elastic foundation [Fig. 1(a)]. To focus on the main issue, only transverse cracks will be considered. Therefore, we can consider a longitudinal strip of a plate having a unit width together with its foundation. Since we are interested only in the average crack spacing, the crack spacing, $2 L$, and the vertical crack depth $a$ are assumed to be uniform. We may expect a periodic solution, and so we can analyze only a cell of length $2 L$, with the crack located in the center [Fig. 1(b)].

Following the analysis of Rice and Levi (1972) and Okamura et al. (1972), the effect of a crack in a plate or beam is represented by an increase in compliance. Let $\Delta_{l}$ and $\theta_{t}$ be the total elongation and relative rotation between the ends of the beam with an edge crack in the center [Fig. 1(b)], loaded by moment $\boldsymbol{M}$ (moment per unit width) and axial tension $N$ (force per unit width) at both ends. The total deformations can be expressed as the sum of deformations, $\Delta_{0}$ and $\theta_{0}$, of the beam 
(a)

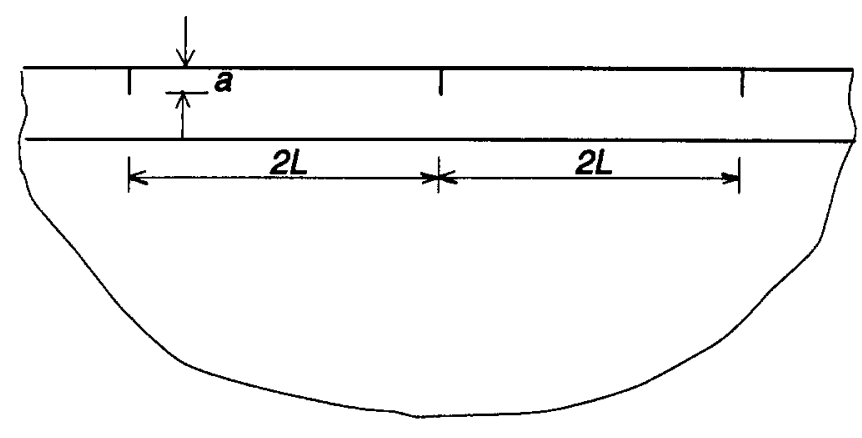

(b)

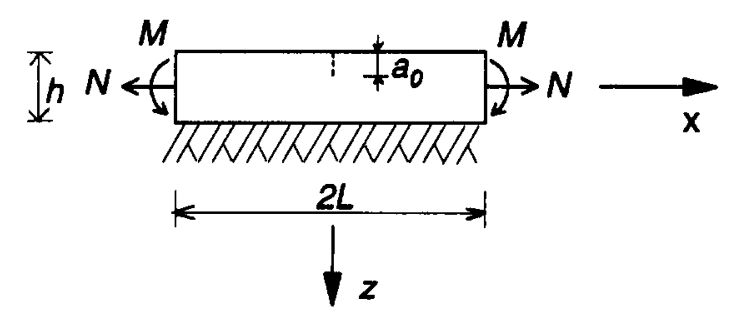

FIG. 1. (a) Pavement of Elastic Foundation; (b) Unit Cell with Edge Crack

without a crack and additional deformations, $\Delta$ and $\theta$, caused by the crack, that is

$$
\Delta_{t}=\Delta_{0}+\Delta ; \quad \theta_{t}=\theta_{0}+\theta
$$

The additional deformations caused by the crack may be expressed as

$$
\theta=\lambda_{M M} M+\lambda_{M N} N ; \quad \Delta=\lambda_{N M} M+\lambda_{N N} N
$$

where $\lambda_{M M}, \lambda_{M N}$, and $\lambda_{N N}=$ compliance functions; $\lambda_{M M}=$ additional rotation due to a crack caused by a unit value of $M$; $\lambda_{N N}=$ additional elongation caused by a unit value of $N$; and $\lambda_{M N}=$ elongation or rotation caused by a unit value of $M$ or $N$, respectively ( $\lambda_{M N}=\lambda_{N M}$ because the plate is linear elastic). The stress intensity factors may be represented as

$$
K_{N}=\frac{N}{\sqrt{h}} k_{N}(\alpha), \quad K_{M}=\frac{M}{h \sqrt{h}} k_{M}(\alpha)
$$

where $\alpha=a / h=$ relative crack length. It is easy to show that the compliance functions can be expressed as

$$
\lambda_{i j}=\frac{2}{h^{m}} \frac{1-v^{2}}{E} \int_{0}^{\alpha} k_{i}\left(\alpha^{\prime}\right) k_{j}\left(\alpha^{\prime}\right) d \alpha^{\prime}
$$

where $i, j=M$ or $N$; and exponent $m=0$ for $\lambda_{N N}, m=1$ for $\lambda_{N M}$, and $m=2$ for $\lambda_{M M}$. The expressions for $\lambda_{M M}$ and $\lambda_{N N}$ as functions of $\alpha$ can be obtained directly from handbooks [e.g., Tada et al. (1985)]. Only $\lambda_{M N}$ as a function of $\alpha$ needs to be obtained from the following equation using numerical integration (see Appendix I).

The differential equation of equilibrium for deflections $w$ of a plate strip resting on a Winkler elastic foundation is

$$
D \frac{d^{4}}{d x^{4}}+k_{v} w=0
$$

where $D=E h^{3} / 12\left(1-v^{2}\right)=$ cylindrical stiffness of the plate; $k_{v}=$ foundation modulus (in the vertical direction); $E=$ Young's modulus of plate; $v=$ Poisson's ratio; and $h=$ plate thickness. This differential equation is the same as that for an elastic beam of unit width except that $E$ is replaced with
$E /\left(1-\nu^{2}\right)$. For the sake of simplicity we consider cracking in the absence of applied load. The compliance of the plate strip can be expressed as follows:

$$
C_{M M}=\frac{2 \theta(L)}{M(L)}=\frac{\Psi(\lambda L)}{\lambda D \Phi(\lambda L)}=\frac{\Omega(\lambda L)}{\lambda D}
$$

where $\lambda^{4}=k_{v} / 4 D$; and $1 / \lambda$ has the dimension of length. The detailed solution is given in Appendix II. Function $\Omega$ is plotted in Fig. 2. As can be seen, when the nondimensional length $\lambda L$ becomes larger than about 1.8 , the compliance function starts to fluctuate and eventually approaches 4 . In other words, the bending compliance will stop increasing with beam length, which is not reasonable.

The assumption that the foundation reaction is proportional to deflection becomes unrealistic if the deflection is upward because the plate separates from the foundation if the tensile reaction of foundation exceeds the own weight of the plate. The total reaction force of the foundation must be nearly zero if there is no applied load, and so the integral of the deflection along the entire beam length must be zero if the foundation force is proportional to deflection. Therefore, a large portion of the beam must deflect upward if the beam is long. A small portion of the beam with upward deflection is acceptable because in reality the own weight keeps the beam in contact with the foundation. But if there are large uplifting portions of the beam, then the assumption of a Winkler foundation becomes inadequate.

Considering a portion of the beam to separate from the foundation would greatly complicate the solution. Therefore, we will consider only the reference case in which the stiffness of foundation is neglected for the whole beam! This case, for which the simple beam theory yields

$$
C_{M M}=\frac{2 \theta(L)}{M(L)}=\frac{2 L}{D}
$$

may be expected to give one bound on the exact solution, and (6) to give the opposite bound. In contrast to (6), compliance (7) depends on the beam length linearly. It will be seen later that bounds obtained from (6) and (7) are very close when the crack spacing is small, but not when it is larger. For large spacing, (7) is on the safe side and thus more reasonable.

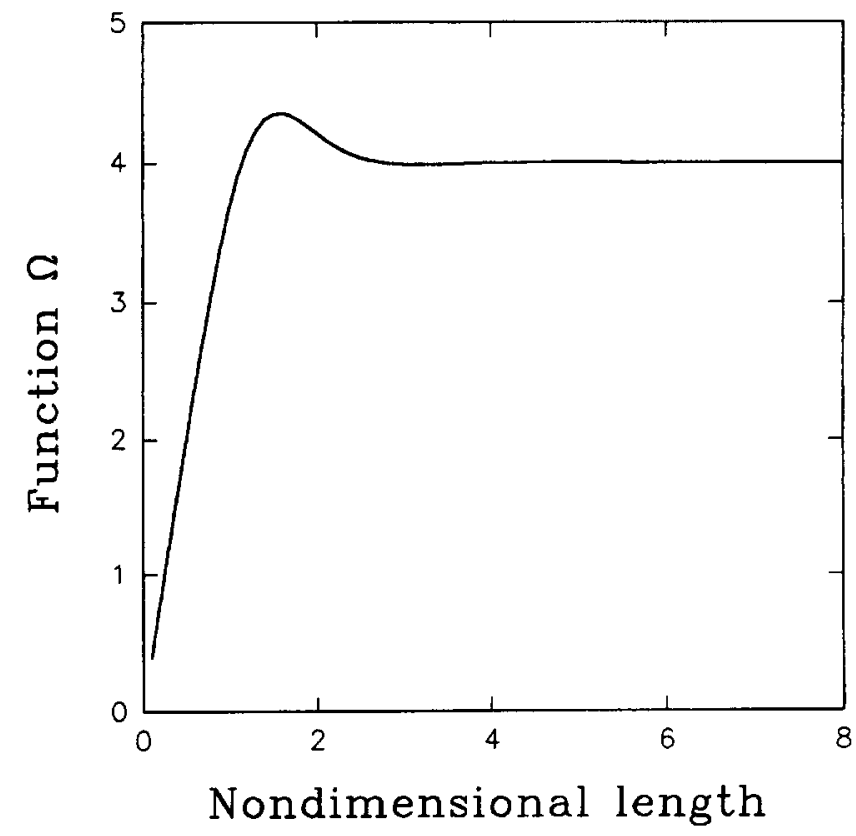

FIG. 2. Bending Compliance Function $\Omega$ of a Beam on Winkler Foundation 
The pavement is stressed by temperature change, which is assumed, for the moment, to vary linearly across the beam thickness. Denote $T_{1}, T_{b}=$ temperatures at the top and bottom of the beam, then

$$
M_{T}=\frac{E \alpha_{T}}{1-\nu} \frac{T_{t}-T_{b}}{2} \frac{h^{2}}{6} ; \quad N_{T}=\frac{E \alpha_{T}}{1-\nu} \frac{T_{t}+T_{b}}{2} h
$$

where $M_{T}$ and $N_{T}=$ thermal moment and tensile force; and $\alpha_{r}=$ thermal expansion coefficient of the pavement material. The rotation due to the combined action of the bending moment and thermal bending can be written as

$$
\theta_{0}=C_{M M}\left(M+M_{T}\right)
$$

where subscript 0 indicates that this is the rotation of the beam without a crack. Since the total rotation at the center of the beam must be zero

$$
\lambda_{M M} M+\lambda_{M N} N+C_{M M}\left(M+M_{T}\right)=0
$$

To complete the formulation, we need to introduce an additional assumption regarding the constraints in the longitudinal direction. Obviously, the constraints depend on the specific structure of the pavement. However, instead of getting into the details of pavement design, we will simply describe a few different mechanical models and postpone the discussion of the relevance and usefulness of these models.

If there is no bond or friction between the pavement and its foundation, and the longitudinal constraint can be neglected, then the axial force $N$ is zero; thus

$$
\left(\lambda_{M M} M+C_{M M}\right)=-C_{M M} M_{T}
$$

If the pavement is restrained from axial contraction, then the axial force is unknown in advance and must be solved from the compatibility condition. The elongation is related to the elastic normal force and the thermal force through the following relation:

$$
\Delta_{0}=C_{N N}\left(N+N_{T}\right) ; \quad C_{N N}=\frac{2\left(1-v^{2}\right) L}{E h}
$$

For this case, one obtains a set of coupled equations

$$
\begin{aligned}
\left(C_{M M}+\lambda_{M M}\right) M+\lambda_{M N} N & =-C_{M M} M_{T} \\
\lambda_{M N} M+\left(C_{N N}+\lambda_{N N}\right) N & =-C_{N N} N_{T}
\end{aligned}
$$

As we will see later, the crack spacing is very sensitive to the axial thermal contractions, which is introduced in (14) by means of $N_{T}$.

The subbases of pavements used many years ago were soft, loose, and without a binder, such as sand, gravel, or clay. Today, stabilizing agents such as cement, lime, or asphalt are added to the subbase materials, which results in a strong bond between the pavement and subbase, opposing relative slip. If the pavement can be assumed to be bonded to the foundation and the tangential force at the bottom can be assumed to be proportional to the horizontal displacement, then the proportionality constant, which is denoted as $k_{h}$ and represents another elastic modulus of the foundation, must also be given. The equilibrium equation for the pavement strip (of unit width) in the axial direction can be expressed as

$$
\frac{E h}{1-v^{2}} \frac{d^{2} u}{d x^{2}}-k_{h} u=0
$$

The solution that satisfies the condition $u(0)=0$ can be written as

$$
u(x)=C \sinh \mu x ; \quad N(x)=\frac{C E \mu h}{1-\nu^{2}} \cosh \mu x
$$

where $C=$ an arbitrary constant and $\mu^{2}=\left(1-v^{2}\right) k_{h} / E h$; and
$2 \pi / \mu=$ wavelength. The compliance function for normal load at the end of the beam is

$$
C_{N N}=\frac{2 u(l)}{N(l)}=\frac{1-v^{2}}{E h \mu} 2 \tanh \mu L
$$

It is straightforward to show that when $k_{h}$ becomes vanishingly small, the compliance function increases and approaches the limit value $C_{N N}=2 l\left(1-v^{2}\right) / E h$. Therefore, the bond compliance reduces to the axial compliance (as intuition suggests). The total bond force must be solved from (13) and (14), with $C_{N N}$ calculated according to (17). The moment contribution of this bond force can be neglected for long beams ( $h / L$ very small).

In the foregoing analysis, the thermal load has been calculated under the assumption that the subbase does not contract or expand with temperature. If a significant temperature change reaches the subbase, the thermal loads defined by (11) must be modified.

\section{CRACK INITIATION THEORY}

The preceding structural analysis allows us to calculate the internal bending moment and axial force once the crack space $2 L$ and crack depth $a$ are known. If there are no initial cracks in the pavement, the internal moment and axial force are a special case of the preceding analysis in which the compliance of the cracked section is let to be zero. As the thermal loading continues to increase, the tensile strength of the pavement is reached and thus one necessary condition for crack initiation becomes satisfied. However, the strength criterion cannot determine what the average crack spacing is, nor can it determine what the average depth of the cracks is. On the other hand, linear elastic fracture mechanics (LEFM) cannot be applied at the moment of initiation, because LEFM considers only the condition of growth of the existing cracks, and because the energy release rate is zero for zero-length cracks. Therefore, one needs additional conditions that govern the process of crack initiation. One of the conditions, expressing the energy balance for finite critical cracks, was introduced and used in an approximate form by Bažant and Ohtsubo (1977) and Bažant et al. (1979). All of the necessary conditions were precisely but briefly formulated by $\mathrm{Li}$ and Bažant (1994) for ice, and later also by $\mathrm{Li}$ et al. (1994) for thermal cracks in a halfplane. They will now be discussed in detail.

The energy release rate can be related to the stress intensity factor $K$ by Irwin's formula $K^{2}\left(1-v^{2}\right) / E$. The total stress intensity factor caused by $M$ and $N$ can be expressed as

$$
K=K_{M}+K_{N} \frac{M}{h \sqrt{h}} k_{M}(\alpha)+\frac{N}{\sqrt{h}} k_{n}(\alpha)
$$

where $N$ and $M$ are determined as already described. Although $k_{N}$ and $k_{M}$ increase with relative crack length $\alpha=a / h, N$ and $M$ decrease with $a / h$ because, if the beam is totally severed, $N$ and $M$ become zero due to the loss of restraint. Once cracks of a certain sufficient length $a>0$ develop, Griffith's criterion of static crack growth, stating that the energy release $G$ must be equal to the fracture energy of the material $G_{f}$, becomes satisfied. During formation of the initial cracks, the total energy needed to form new crack surfaces, which is $a G_{f}$ for each crack and equals the shaded area under the curve in Fig. 3(a), must be equal to the energy released by the structure. This is the very condition that determines the crack spacing.

The process of initial crack formation consists of a transition from the preinitiation state, at which the strength criterion is satisfied, to the postinitiation state, at which Griffith's criterion is satisfied. The complete crack initiation theory proposed in $\mathrm{Li}$ and Bažant (1994) consists of the following three conditions:

JOURNAL OF ENGINEERING MECHANICS / MARCH 1997 / 269 

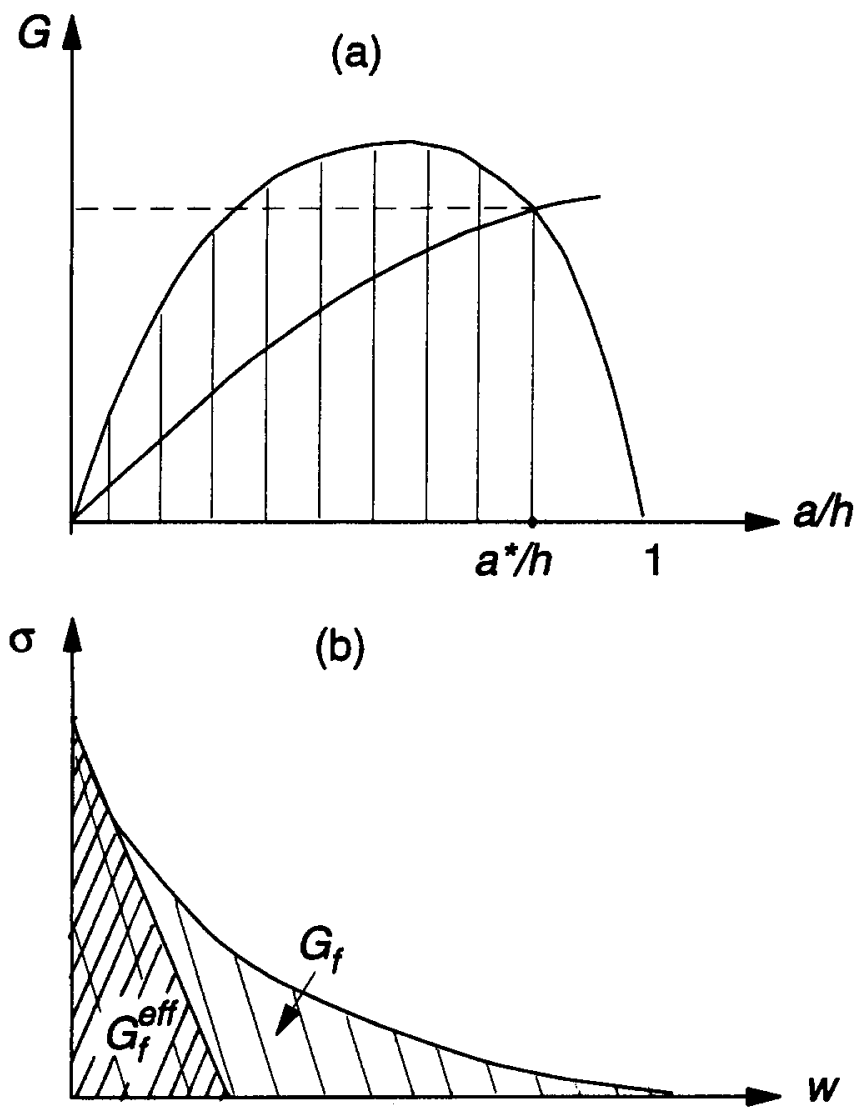

FIG. 3. (a) Schema of Crack Initiation Theory; (b) Measuring of Effective and Total Fracture Energies

1. The maximum tensile stress $\sigma_{\max }$ before the initial cracks form must be equal to the tensile strength $f_{t}^{\prime}$ of the pavement in the preinitiation state.

2. The energy release rate $G$ of the structure after the initial cracks form must be equal to the fracture energy $G_{f}$ of the pavement material in the postinitiation state.

3. The energy must be conserved during the crack initiation process, i.e., the total release of strain energy must be equal to the energy $a G_{f}$ per crack, required to create the crack surfaces.

Conditions 1 and 3 were also implicit to the initial crack spacing formula proposed by Bažant and Ohtsubo (1977) and Bažant et al. (1979), but condition 2 was missing (causing the initial crack length to be left unspecified and assumed empirically). Condition 3 was mathematically expressed only in an approximate manner.

The foregoing three conditions play different roles in the solution procedure. The first condition decides the critical value of the temperature differential, but does not affect the initial crack length and spacing. This yields the temperature profile, which is then considered fixed during the crack initiation process. The axial force $N$ and loading moment $M$ can then be solved as functions of crack depth $a$ and half-spacing $l$.

The second and third conditions must be solved together to determine the crack spacing $l$ and crack depth $a$ for the postinitiation state. These conditions read

$$
G(a)=G_{f} ; \quad \Pi^{*}(0)-\Pi^{*}(a)=a G_{f}
$$

where $\Pi *(a)=$ complementary elastic strain energy of the structure as a function of crack length. According to the definition of the energy release rate, $(19 b)$ can also be expressed as

$$
\int_{0}^{a} G\left(a^{\prime}\right) d a^{\prime}=a G_{f}
$$

or $\bar{G}=G_{f}$ where $\bar{G}=\int_{0}^{a} G\left(a^{\prime}\right) d a^{\prime} / a=$ average energy release rate for crack length $0 \leq a^{\prime} \leq a$. Usually the integral in (20) must be evaluated by numerical quadrature.

In the preceding analysis, the material is assumed to be perfectly brittle, i.e., to follow LEFM. Perfect brittleness means that the fracture process zone around the crack tip is negligible compared to the characteristic structural dimension. Portland cement concrete, of course, is not perfectly brittle but quasibrittle. Its fracture process zone is typically $2-20$ in. long (BaŽant 1986; Planas and Elices 1991). To take the nonlinear behavior in the process zone into consideration, one must perform fracture mechanics analysis, which can be done, for instance, according to the cohesive (or fictitious) crack model (Hillerborg et al. 1976) in which the cohesive stress is a function of the crack opening displacement or, equivalently, according to the crack band model (Bažant and Oh 1983).

In a realistic description, diffuse microcracking gradually localizes during the crack initiation process into a discrete microcrack. The energy dissipated during this transition is underestimated by (20). A more accurate description of the localization could be obtained with a nonlocal damage model. An easier approach using nonlinear fracture mechanics would also be too complicated for the present purpose and must be relegated to a future study.

A simple practical solution can be obtained by distinguishing different values of fracture energy, $G_{f}$ and $G_{f}^{i n}$. It is known that the fracture energy of concrete, $G_{f}$, determined by the work-of-fracture method introduced for concrete by Hillerborg (1985), which typically is about $80-120 \mathrm{~J} / \mathrm{m}^{2}$, is larger than the fracture energy $G_{f}^{\text {in }}$ obtained by either the size effect method (Bažant and Pfeiffer 1987) or the two-parameter model of Jenq and Shah (1985), which typically is about $30-60 \mathrm{~J} / \mathrm{m}^{2}$ [see also Bažant (1996)]. The reason can be explained by considering the softening stress-displacement curve [Fig. 3(b)] of the cohesive (or fictitious) crack model.

Except for impracticably large specimens, the maximum load of notched fracture specimens as well as unnotched specimens is reached very early in the fracture process, while the opening of the cohesive crack is everywhere still quite small. This means that what matters for crack initiation is only the initial tangent of the softening stress displacement curve in Fig. 3(b), and thus the area under this tangent, representing what may be called the initial fracture energy, $G_{f}^{i n}$. On the other hand, in the work-of-fracture method, the fracture energy is determined from the energy required for a complete break of the specimen. This means that, at each point of the break, the dissipated energy equals the entire area under the stress-displacement curve with its long tail [Fig. 3(b)], which represents the total fracture energy $G_{f}$.

From this explanation, it is clear that the fracture energy to be considered for the formation of the initial cracks in the pavement should be approximately $G_{f}^{i n}$. On the other hand, the fracture energy to be considered for the subsequent growth of deep cracks should be closer to $G_{f}$, which is about twice as large. In this light, the second condition of crack formation may be written as

$$
G=G_{f}^{\text {in }} \quad \text { with } \quad G_{f}^{i n}=\beta G_{f}
$$

The typical value of $\beta$ is about $1 / 2$ (Bažant 1996) and can be as small as $1 / 3$. The third condition, as written in (19a), uses the total fracture energy $G_{f}$.

As will be seen later, the influence of $\beta$ on the final crack spacing and crack depth is not very important when the $G_{f}$ value is kept constant. However, the final solution depends significantly on the $G_{f}$ value. In other words, $G_{f}$ is the more important quantity for pavement characterization.

For asphalt concrete, the difference between $G_{f}^{\text {in }}$ and $G_{f}$ is probably even larger than for Portland cement concrete, as 
suggested by data on crack spacing. It is essential to characterize the material in a proper way.

\section{NUMERICAL METHOD}

To understand the dependence of the solution on various material properties, it is useful to introduce dimensionless nominal stresses

$$
\sigma_{N}=\frac{N}{f_{i} h} ; \quad \sigma_{M}=\frac{6 M}{f_{t} h^{2}}
$$

where $f_{t}=$ tensile strength of the material. With these notations, the first condition of the crack initiation theory can then be simply expressed as

$$
\sigma_{M}+\sigma_{N}=1
$$

while the second condition becomes

$$
\left[\sigma_{N} k_{N}(\alpha)+\frac{\sigma_{M}}{6} k_{M}(\alpha)\right]^{2}=\frac{\beta l_{0}}{h}
$$

where $l_{0}=E G_{f} /\left(1-\nu^{2}\right) f_{1}^{2}=$ material length of the pavement. The third condition becomes

$$
\frac{1}{\alpha} \int_{0}^{\alpha}\left[\sigma_{N} k_{N}\left(\alpha^{\prime}\right)+\frac{\sigma_{M}}{6} k_{M}\left(\alpha^{\prime}\right)\right]^{2} d \alpha^{\prime}=\frac{l_{0}}{h}
$$

Although not explicitly written in (24) and (25), the dependence of $\sigma_{N}$ and $\delta_{M}$ on the relative crack depth as well as the crack spacing comes indirectly through the solution of the fracture problem. The dimensionless equations for the nominal stresses characterizing the internal forces can now be written as

$$
\begin{aligned}
& \left(C_{M M}^{*}+\lambda_{M M}^{*}\right) \sigma_{M}+\lambda_{M N}^{*} \sigma_{N}=-C_{M M}^{*} \sigma_{M}^{T} \\
& \lambda_{M N}^{*} \sigma_{M} / 6+\left(C_{N N}^{*}+\lambda_{N N}^{*}\right) \sigma_{N}=-C_{N N}^{*} \sigma_{N}^{T}
\end{aligned}
$$

where $\sigma_{M}^{T}$ and $\sigma_{N}^{T}=$ dimensionless nominal stresses of the thermal moment and thermal tensile force defined in (8) and normalized in the same way as (22). Eq. (27) should be disregarded if the axial constraint is not considered.

The dimensionless compliances due to crack are defined as

$$
\lambda_{N N}^{*}=\frac{E}{1-v^{2}} \lambda_{N N} ; \quad \lambda_{M N}^{*}=\frac{E h}{1-v^{2}} \lambda_{M N} ; \quad \lambda_{M M}^{*}=\frac{E h^{2}}{6\left(1-v^{2}\right)} \lambda_{M M}
$$

and their closed-form expressions are given in Appendix $\mathbf{I}$. The dimensionless compliance functions are defined as

$$
C_{N N}^{*}=\frac{E}{1-v^{2}} C_{N N} ; \quad C_{M M}^{*}=\frac{E h^{2}}{6\left(1-v^{2}\right)} C_{M M}
$$

Since the compliance function due to a crack is zero if the crack length is zero, the nominal stresses in the preinitiation state can be simply solved as $\sigma_{M}=-\sigma_{M}^{T}$ and $\sigma_{N}=-\sigma_{N}^{T}$. The first condition of the initiation theory, therefore, becomes

$$
\sigma_{M}^{T}+\sigma_{N}^{T}=-1
$$

which becomes a condition to determine the critical temperature differential. When the distribution of the thermal stress is far from linear, then (30) should be replaced by the condition in (23).

Combining (24) and (25), we obtain

$$
\begin{gathered}
\frac{\beta}{\alpha} \int_{0}^{\alpha}\left[\alpha_{N} k_{N}\left(\alpha^{\prime}\right)+\frac{\sigma_{M}}{6} k_{M}\left(\alpha^{\prime}\right)\right]^{2} d \alpha^{\prime} \\
-\left[\sigma_{N} k_{N}(\alpha)+\frac{\sigma_{M}}{6} k_{M}(\alpha)\right]^{2}=0
\end{gathered}
$$

For a given value of crack spacing $2 l$, the value of $\alpha$ can be solved from (31). For this purpose one needs to note that the net stress intensity factor $K=0$ when $\alpha \rightarrow 0$ (because the stress intensity factor is generally known to approach zero as $\alpha^{1 / 2}$ when $\alpha \rightarrow 0$ ) and that $K=0$ when $\alpha \rightarrow 1$ because the compliance function due to crack is generally known to approach infinity as $1 /(1-\alpha)^{2}$, forcing the nominal stresses due to tension or bending to approach zero as $(1-\alpha)^{2}$. Thus, even though the stress intensity factor due to unit load approaches infinity as $(1-\alpha)^{-3 / 2}$, the net stress intensity factor $K$ must approach zero as $(1-\alpha)^{1 / 2}$ when $\alpha \rightarrow 1$. In terms of the energy release rate $G, K$ approaches zero as $1-\alpha$ when $\alpha$ approaches 1 .

The intermediate variation of $K$ is more complex. Depending on the tensile component of thermal stress, $\sigma_{N}^{T}, K$ can be negative for sufficiently long cracks, as shown in Fig. 4 (where negative $K$ values would be those under the horizontal plane). The thermal bending stress $\sigma_{M}^{T}$ is determined by (30). This phenomenon is particularly conspicuous for large compressive axial thermal stress $\sigma_{N}^{T}$ (negative values in the plot). Although $K$ becomes positive again for even larger crack lengths, such lengths cannot be reached unless additional energy is provided to assist the crack to jump through the valley of negative $K$. Although the energy release rate is still positive for negative $K$, this value does not represent the energy that can be released from the structure by crack propagation. For this reason, it is necessary to ensure, by means of a proper numerical algorithm, that the solution of crack depth does not cross the valley of negative $K$ values.

Based on these observations, we know that $G$ must be an increasing function initially and a decreasing function eventually. When $G$ is an increasing function, its average value from 0 to $\alpha$ must be smaller than the current value. Since $\beta$ is less than 1, the left-hand side of (31) must be negative for a small $\alpha$ value. For a large $\alpha$ value, the average energy release rate must be a positive number while the energy release rate approaches zero, and so the left-hand side of (31) must be positive. Consequently, between 0 and the boundary of negative $K$ values, there must be a relative crack length $\alpha$ that satisfies (31). This property is essential for designing a successful numerical algorithm to solve the unknown crack spacing and crack depth. Once the crack depth has been solved as a function of crack spacing, one can use (24) to determine the

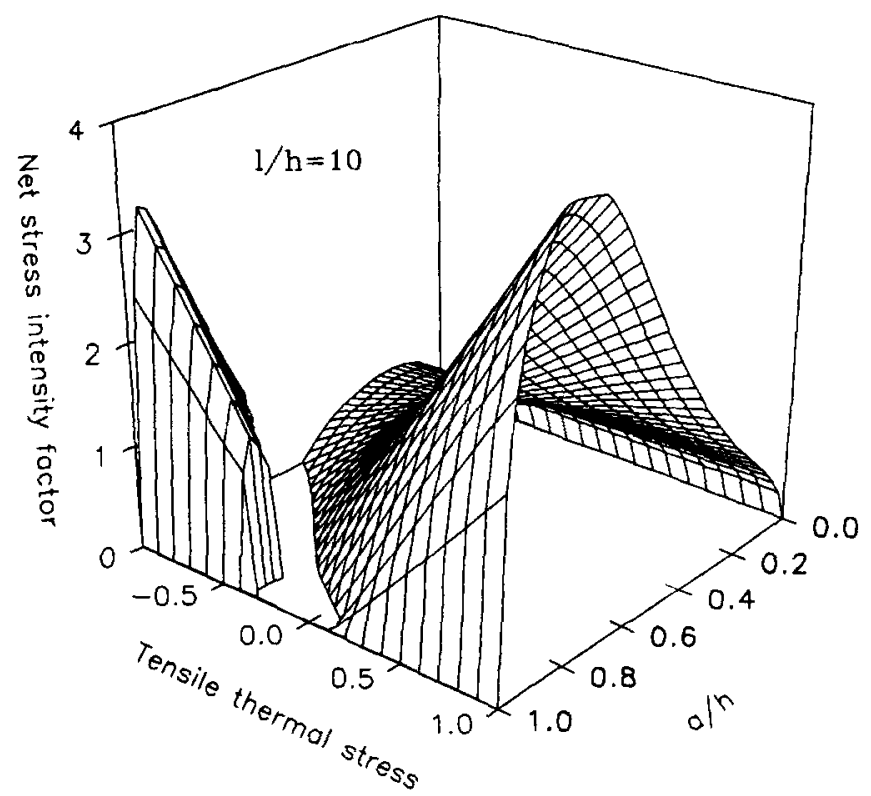

FIG. 4. Net Stress Intensity Factor as a Function of Crack Length and Loading Configuration

JOURNAL OF ENGINEERING MECHANICS / MARCH 1997/ 271 
crack spacing. This is the main feature of the present numerical method.

\section{GENERAL BEHAVIOR OF THE MODEL}

In the following numerical examples, the thickness of the concrete slab is taken as $10 \mathrm{in}$. $(25.4 \mathrm{~cm})$, which is a typical value in pavement design. The material properties used in these calculations are $E=4.2 \times 10^{6} \mathrm{psi}(28.97 \mathrm{GPa}), \nu=0.18$, and $k_{v}=400 \mathrm{lb} / \mathrm{in}^{3}(108.7 \mathrm{MPa} / \mathrm{m})$. With these parameters, $\lambda=0.0293 /$ in. $(1.25 / \mathrm{m})$. Parameter $\beta$ is chosen to be $1 / 3$. For typical portland cement concretes the material length is about 7 in. $(17.78 \mathrm{~cm}$ ), provided the fracture energy (or the corresponding toughness) of concrete is defined by the work-offracture method. The tensile strength $f_{t}$ and thermal expansion coefficient $\alpha_{T}$ need not be specified at this point because these parameters do not enter into (24) or (25). They will determine the critical value of the temperature differential.

The simplest case is that of pure bending without axial constraints. The crack spacing and crack depth are plotted in Figs. 5(a) and 5(b) as functions of the material length $l_{0}=$ $E G_{f} / f_{t}^{\prime 2}$. The effect of different compliance functions is clearly demonstrated. If $C_{M M}$ is defined by (6), that is, if the pavement is modeled as a beam resting on a Winkler foundation, then there is an upper limit for $l_{0}$ beyond which there is no solution for the crack initiation problem. This upper limit is obviously related to the upper bound on the compliance function, as explained in the previous discussion. On the other hand, the crack spacing is basically a linear function if $C_{M M}$ is defined by (7), which appears more reasonable from the physical point of view. When $l_{0}$ is small, both compliance functions yield similar results.
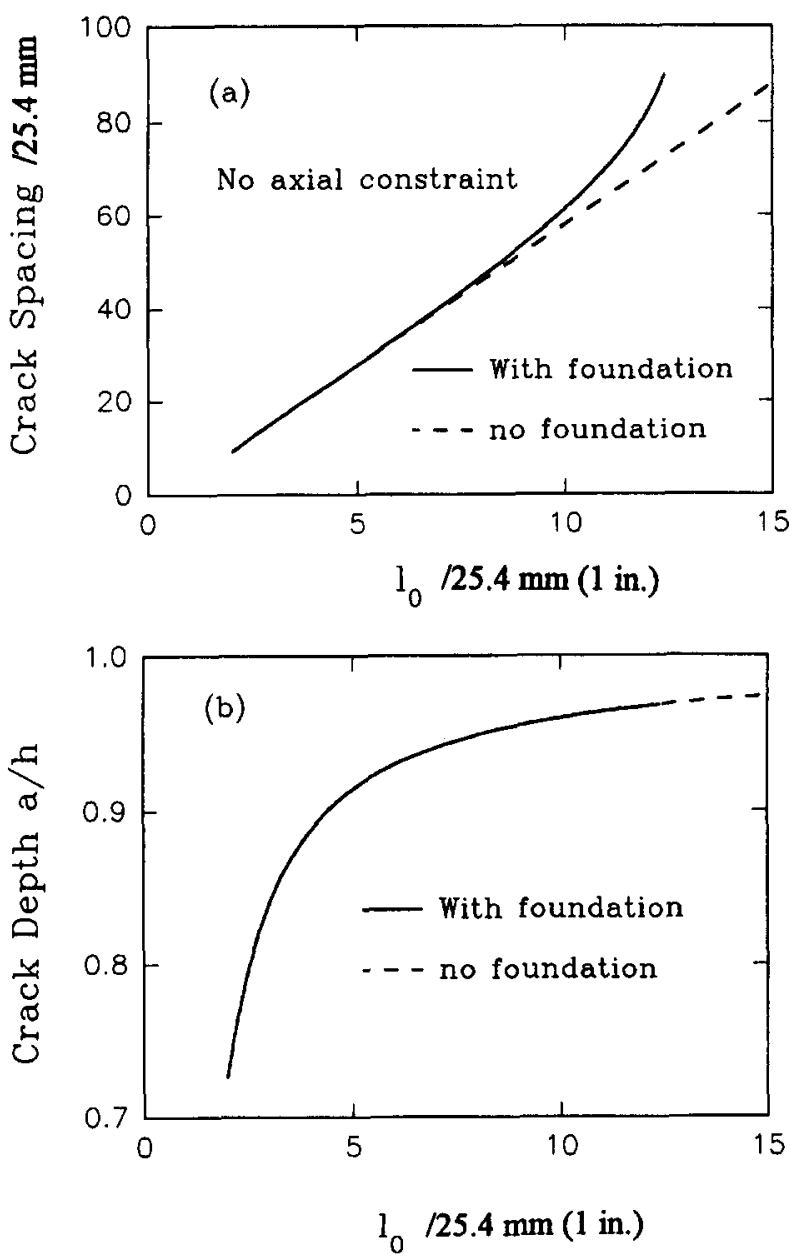

When the axial deformation is restrained, the crack opening is limited by the compressive force it generates and the crack depth is smaller, whereas the crack spacing is larger for the same $l_{0}$, as seen from Figs. 5(c) and 5(d). The crack spacing approaches infinity when $l_{0}$ approaches a certain finite value. One might argue that the extreme sensitivity of the crack spacing when $l_{0}$ is close to its upper limit value might be unreasonable from the physical point of view. For small $l_{0}$ values, these two compliance functions again yield similar results. Therefore, only the compliance function defined in (7) will be used in further considerations.

The effect of bond between the pavement and the foundation is shown in Fig. 6(a) with $l_{0}$ fixed as 3, 7, and 12 in. $(7.62,17.78$, and $30.48 \mathrm{~cm})$, respectively. Adequate data for the $k_{h}$ value are difficult to find in the open literature. If we assume $k_{h}=k_{v}$, then $\mu \approx 3 \times 10^{-3} / \mathrm{in}$. $(0.117 / \mathrm{m})$. At this magnitude, the shear bond is not particularly important if $l_{0}=$ 7 in. $(17.78 \mathrm{~cm})$. For larger values of $l_{0}$, though, the shear bond can be important. For smaller values of $l_{0}$, the shear bond effect can be neglected for all practical purposes.

The effect of $\beta$ is shown in Fig. $6(\mathrm{~b})$ for the case of no shear bond but constrained axial deformation. Although it may come as a surprise, the influence of $\beta$ is not important at all. The main influence on crack spacing, in addition to the material length $l_{0}$, comes from the thermal stress distribution. As shown in Fig. 6(c), if there is substantial tension due to the thermal loading, then the spacing is significantly reduced. If there is compression, the spacing is dramatically increased.

It is instructive to know what will happen when there is only a uniform temperature drop while the axial deformation is constrained. Since there is no bending, the beam would sim-
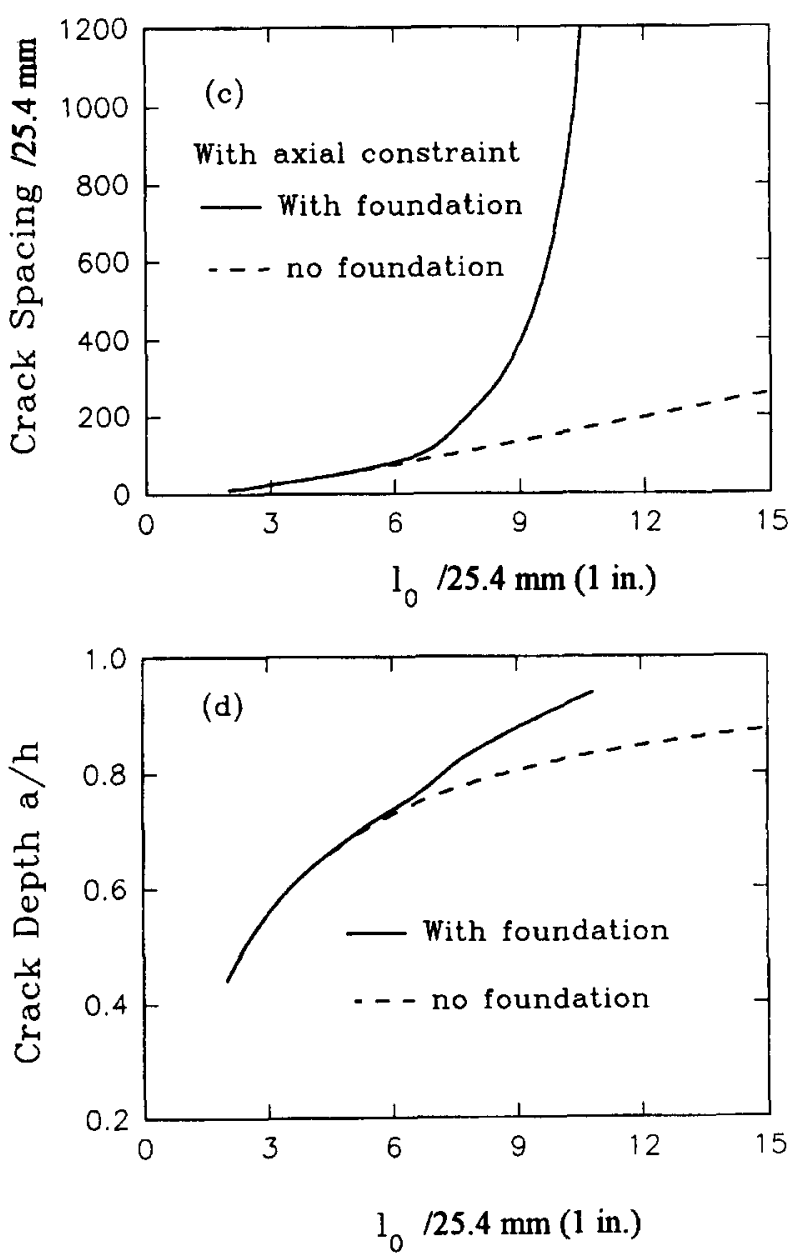

FIG. 5. Crack Spacing and Depth as a Function of $b_{0}:(a, b)$ with No Axial Constraint; $(c, d)$ with Axial Constraint 

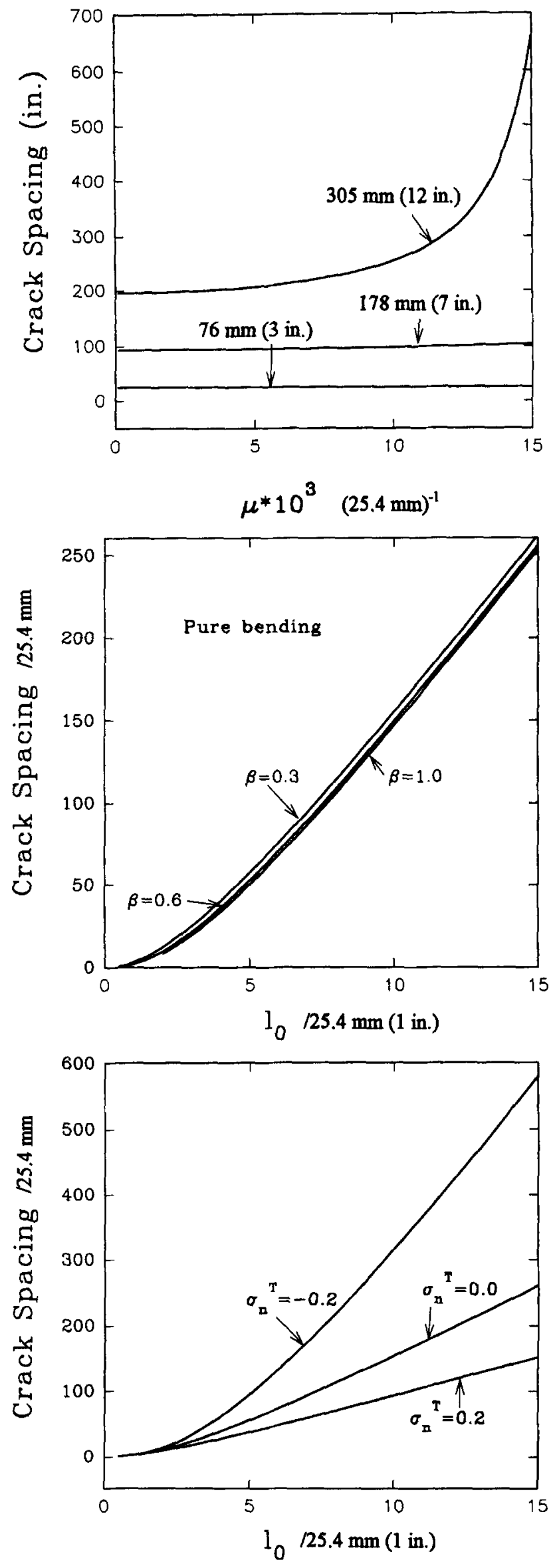

FIG. 6. Crack Spacing as a Function of: (a) Tangential Bond $\mu$; (b) Nonlinearlty Coofficient B; (c) Axial Thermal Stress ply break into segments. The average length of these segments in this limit case would be the crack spacing. Because the beam is totally severed, the crack depth $a=h$, the beam depth. Therefore the second condition is nullified, and only the first and the third conditions remain. The third condition can be written as $2 L h s \sigma^{2} / 2 E^{\prime}=h G_{f}$. The conversion must occur at $\sigma=f_{t}$. As a result, one finds that $2 L=2 l_{0}$. This analysis shows that $l_{0}$ is simply the ratio of the specific elastic strain energy to the fracture energy, which is why $l_{0}$ is one of the main factors that determine the crack spacing. This minimum value of crack spacing was also obtained by $\mathrm{Li}$ et al. (1995) in their study of spacing of parallel cracks initiated from the surface of an elastic half plane.

For more general loading, the strain energy that can be stored, as well as the fracture energy needed, depend on the crack depth. This is why the second condition of the initiation theory must be employed. However, the main picture is still the global energy conservation. The more strain energy stored per unit length, the smaller the crack spacing will be, and vice versa. For instance, the strain energy per unit length becomes smaller if the restraint by the foundation is considered. That is why the spacing predicted with the Winkler foundation is generally larger than the spacing without any foundation. Likewise, the axial constraint causes the crack spacing to be larger because less elastic strain energy is stored in the beam per unit length. The effect of axial thermal loading can also be explained. As can be seen from Fig. 4, with a uniform thermal contraction, the overall $K$ value (and thus the total energy that can be released from the structure) increases; thus the crack spacing is smaller. On the other hand, with uniform thermal expansion, the energy that can be released from the structure becomes smaller, and thus the crack spacing is larger.

\section{EFFECT OF NONLINEAR TEMPERATURE DISTRIBUTION}

As shown by Armaghani et al. (1987), the nonlinear temperature distribution caused by daily temperature fluctuation is not very significant. In other words, the thermal load calculated according to (8) is a good approximation if we are concerned only with the thermal stress caused by daily temperatures changes. However, adequately designed pavements should not develop cracks under normal daily temperature changes. It is likely that the critical condition is met when drastic cooling occurs, for instance, in a summer rainstorm [Fig. 7(a)]. The temperature distribution becomes very different from a steady-state (linear) distribution, and so the thermal loads calculated according to (8) are no longer realistic. Asphalt concrete pavements invariably develop thermal cracks in the northern states of the United States and in Canada during the winter season when the temperature drops dramatically at night, producing the temperature profile schematically plotted in Fig. 7(b). In these cases, (8) must be modified.

Let $T(z)$ be the distribution of temperature increment (that is, the measured temperature minus the reference temperature at which there is no thermal stress) across the pavement thickness. The thermal stress distribution is $E \alpha_{T} T(z) /(1-v)$. The critical temperature distribution is determined by the condition that the stress at the top surface must be equal to the tensile strength of the material. The thermal bending moment and thermal tensile force are defined as

$$
M_{T}=\frac{E \alpha_{T}}{1-v} \int_{-h / 2}^{h / 2} T(z) z d z ; \quad N_{T}=\frac{E \alpha_{T}}{1-v} \int_{-h / 2}^{h / 2} T(z) z d z
$$

The dimensionless nominal thermal stresses $\sigma_{M}^{T}$ and $\sigma_{N}^{T}$ are, again, defined according to (22). However, the crack initiation condition (30) for the nominal thermal stresses, which has been established under the assumption of a linear temperature profile, must be discarded. 
(a)

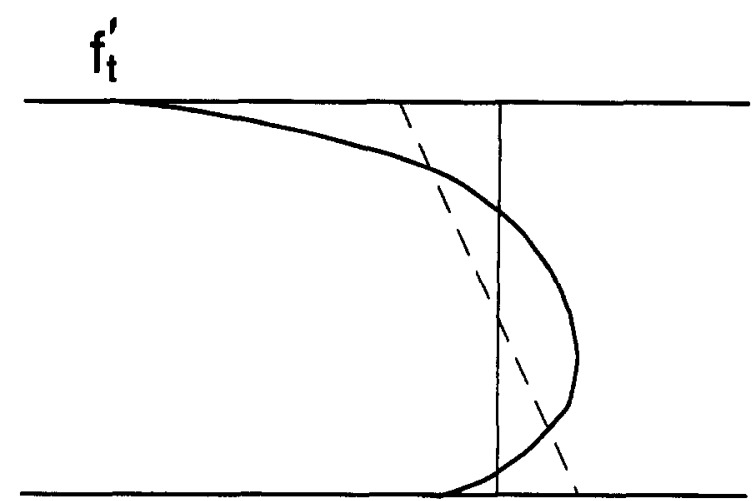

(b)

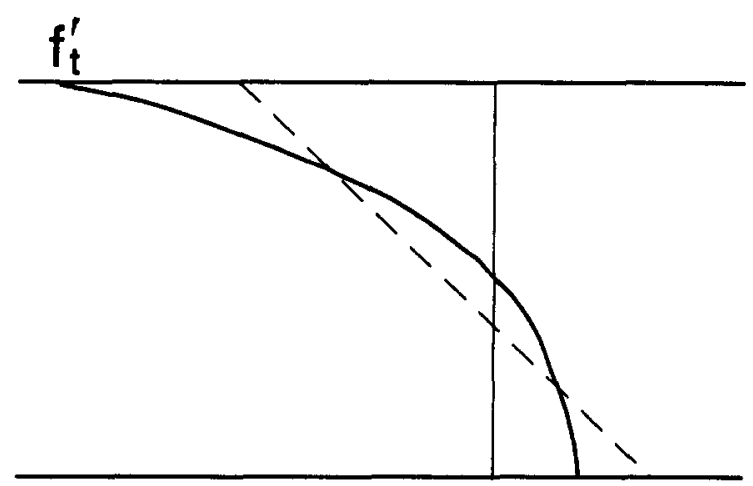

FIG. 7. Schema of Nonlinear Distrlbutions of Thermal Stress

It is assumed that the elastic bending and tensile stresses can still be determined by (26) and (27) during crack propagation. This is because, once the cracks occur, the deformation is dominated by bending and tension further away from the cracked cross section. As a result, cracks are initiated with a lower level of strain energy stored per unit length of pavement, which in turn causes larger crack spacing. This effect can be important when theoretical predictions are to be compared with field observations.

As an example, let us estimate the crack spacing in a typical asphalt concrete pavement in Illinois. The crack spacing of about 8-12 $\mathrm{m}$ on the average has been observed after the first winter. Similar values of the first-year crack spacing can also be deduced from the data of McLeod [in the discussion of a paper by Ruth et al. (1982)]. The properties of asphalt concrete vary strongly with temperature. Asphalt concrete becomes quite brittle at low temperatures, especially below the freezing point. Although experimental data on material length $l_{0}$ are not available, we may reasonably assume $l_{0}=10 \mathrm{in}$. $(25.4 \mathrm{~cm})$ for asphalt concrete at low temperature, judging from the fact that portland cement concrete typically has $l_{0}=7$ in. $(17.78 \mathrm{~cm})$. Assume also that the critical thermal stress $\sigma_{M}^{T}=-0.7$ (dimensionless value) and $\sigma_{N}^{T}=0$ during a rapid temperature drop in a winter night. Furthermore, it is assumed that the pavement thickness is $10 \mathrm{in} .(25.4 \mathrm{~cm})$ and the effect of foundation is neglected [i.e., the elastic bending compliance is calculated according to (7), and the shear bond between the pavement and its subbase is considered]. Under these assumptions, the calculated crack spacing is $2 l=388 \mathrm{in}$. ( $\cong 9.86 \mathrm{~m}$ ). This value is realistic in comparison with field observations. If the nonlinear critical temperature yields $\sigma_{M}^{T}=-0.5$, then the crack spacing would become more than 800 in. $(20.3 \mathrm{~m})$, which may still be reasonable.

For more reliable estimates, one must resort to the actual temperature variation data for a specific locality and solve the temperature profile $T(z)$ from the equation of thermal conduction. In addition, heavy traffic loads, although not considered in this paper, doubtless also contribute to crack initiation, thus lowering the critical values of thermal stresses.

\section{MAIN OBSERVATIONS AND CONCLUSIONS}

1. The crack spacing in pavements can be influenced by many factors. The most important are the material length $l_{0}=E G_{f} / f_{t}^{\prime 2}$ and the distribution of thermal stress. The foundation stiffness can be important only under unusual conditions, such as a strong bond.

2. For quasi-brittle materials, there are three conditions that govern crack initiation and spacing: (1) the maximum tensile stress before the cracks form must be equal to the tensile strength of the pavement; (2) the energy release rate after the initial cracks have formed must be equal to the fracture energy; and (3) the total strain energy release must be equal to the total energy required to form the initial cracks.

3. A distinction must be made among the values to be used for fracture energy. The initial fracture energy, which corresponds to the area under the initial tangent of the stress displacement curve in the cohesive (fictitious) crack model and is easily determined from the size effect in maximum load tests, can be about $1 / 2$ or perhaps as little as $1 / 3$ of the total fracture energy, which is defined by the work-of-fracture method and corresponds to the total area under the aforementioned curve, including its long tail. The initial fracture energy should be used in the second condition of crack initiation theory, while the total fracture energy should be used in the third condition.

4. The main principle that controls crack spacing is energy conservation, as suggested already by Bažant and Ohtsubo (1977). If the strain energy stored per unit length of pavement that can be released by crack formation and growth is large, then the crack spacing is small, with $2 l_{0}$ being the smallest possible value.

5. Thermal cracking usually occurs during extreme weather conditions, in which the thermal stress distribution across the pavement thickness can be far from linear. The cracks can then initiate under rather small stress levels and with a large crack spacing.

\section{ACKNOWLEDGMENTS}

The first writer wishes to thank the Department of Civil Engineering at The University of Akron for granting an assistantship before and partly during her work on this study at Northwestern University as predoctoral visiting fellow. Further support covering her postdoctoral appointment at Northwestern University as well as the work of the second and third writers was received partly under NSF Grant MSS-911-4626 to Northwestern University, and partly from the Center for Advanced Cement Based Materials at Northwestern University.

\section{APPENDIX I. STRESS INTENSITY FACTORS AND COMPLIANCE FUNCTIONS}

According to Tada et al. (1985), with $\xi=a / h$,

$$
\begin{gathered}
K_{N}=\frac{N}{\sqrt{h}} k_{N}\left(\frac{a}{h}\right) \\
k_{N}(\xi)=\sqrt{2 \tan \frac{\pi \xi}{2}} \frac{0.752+2.02 \xi+0.37[1-\sin (\pi \xi / 2)]^{3}}{\cos (\pi \xi / 2)} \\
k_{M}(\xi)=6 \sqrt{2 \tan \frac{\pi \xi}{2}} \frac{0.923+0.199[1-\sin (\pi \xi / 2)]^{3}}{\cos (\pi \xi / 2)} k_{M}\left(\frac{a}{h}\right)
\end{gathered}
$$

The compliance function can be calculated according to these 
formulas using numerical integration. Simpson's rule, with subdivision of integral domain [0,1] into 200 intervals, has been used. The resulting formulas, obtained by optimal fitting, are

$$
\lambda_{N N}^{*}(\xi)=\frac{4 \xi^{2}}{(1-\xi)^{2}}\left[0.988-\xi(1-\xi)\left(1.504-1.654 \xi+0.920 \xi^{2}\right)\right]
$$

$$
\lambda_{M M}^{*}(\xi)=\frac{24 \xi^{2}}{(1-\xi)^{2}(1+2 \xi)^{2}}[0.988+\xi(1-\xi)(0.413+0.282 \xi)]
$$

$$
\begin{aligned}
& \lambda_{M M}^{*}(\xi)=\frac{24 \xi^{2}}{(1-\xi)^{2}(1+2 \xi)} \\
& \quad \cdot\left[0.988-\xi(1-\xi)\left(0.578-0.689 \xi+0.196 \xi^{2}\right)\right]
\end{aligned}
$$

Eqs. (35) and (36) are similar to the approximate formulas in the Tada et al. (1985) handbook. Although (35) and (36) are visually undistinguishable from these formulas, they are more accurate. Tada's formulas read

$$
\begin{aligned}
\lambda_{N N}^{*}(\xi) & =\frac{4 \xi^{2}}{(1-\xi)^{2}}\left[0.99-\xi(1-\xi)\left(1.3-1.2 \xi+0.7 \xi^{2}\right)\right] \\
\lambda_{M M}^{*}(\xi) & =\frac{4 \xi^{2}}{(1-\xi)^{2}} \\
\cdot & {\left[5.93-\xi\left(19.69-37.14 \xi+35.84 \xi^{2}+13.12 \xi^{3}\right)\right] }
\end{aligned}
$$

For the mixed compliance function, there is no expression listed in the handbook. The forms of expressions (35), (36), and (37) guarantee the correct asymptotic limits for both $\xi \rightarrow$ $0^{+}$and $\xi \rightarrow 1$.

\section{APPENDIX II. COMPLIANCE FUNCTIONS FOR A BEAM ON WINKLER FOUNDATION}

Due to symmetry, only one half of the beam needs to be considered [Fig. 1(b)]. The elastic solution can be written in the form

$w(x)=e^{\lambda x}\left(C_{1} \cos \lambda x+C_{2} \sin \lambda x\right)+e^{-\lambda x}\left(C_{3} \cos \lambda x+C_{4} \sin \lambda x\right)$

where $\lambda^{4}=k_{v} / 4 D$. The constants $C_{1}, C_{2}, C_{3}$, and $C_{4}$ are determined by the boundary conditions $\theta(0)=V(0)=V(L)=0$, and the solution becomes

$$
\theta(l) \frac{d w(L)}{d x}=2 C_{2} \lambda \Psi(\lambda L) ; \quad M(L)=D \frac{d^{2} w(L)}{d x^{2}}=4 D C_{2} \lambda^{2} \Phi(\lambda L)
$$

where $\Phi$ and $\Psi$ are dimensionless functions given as

$$
\Phi(x)=\cos x \cosh x-Q(x) \sin x \sinh x
$$

$\Psi(x)=Q(x)(\cos x \sinh x-\sin x \cosh x)$

$$
+\cos x \sinh x+\sin x \cosh x
$$

$$
Q(x)=\frac{\cos x \sinh x-\sin x \cosh x}{\cos x \sinh x+\sin x \cosh x}
$$

The rotational compliance of the beam is

$$
C_{M M}=\frac{2 \theta(L)}{M(L)}=\frac{\Psi(\lambda L)}{\lambda D \Phi(\lambda L)}=\frac{\Omega(\lambda L)}{\lambda D}
$$

A factor of 2 is introduced because the rotation at the end of the beam corresponds to one half of the total rotation in the cracked cross section.

\section{APPENDIX III. REFERENCES}

Armaghani, J. M., Larsen, T. J., and Smith, L. L. (1987). “'Temperature response of concrete pavements." Transp. Res. Rec. 1121, Transp. Res. Board, Washington, D.C., 23-33.

Bažant Z. P. (1986). "Mechanics of distributed cracking." Appl. Mech. Review, 39, 675-705.

Bažant Z. P. (1996a). "Analysis of work-of-fracture method for measuring fracture energy of concrete." J. Engrg. Mech., ASCE, 122(2), $138-144$.

Bažant Z. P. (1996b). "Size effect aspects of measurement of fracture characteristics of quasibrittle material." Fracture mechanics of concrete structures, Vol. 3, Aedificatio Publishers, Freiburg, Germany, $1749-1772$

Bažant Z. P., and Cedolin, L. (1991). Stability of structures: elastic, inelastic, fracture and damage theories. Oxford University Press, New York, N.Y.

Bažant Z. P., and Oh, B. H. (1983). "Crack band theory for fracture of concrete." Mat. and Struct., 16, 155-177.

Bažant Z. P., and Ohtsubo, H. (1977). "Stability conditions for propagation of a system of cracks in a brittle solid." Mech. Res. Communications, 4(5), 353-366.

Bažant Z. P., Ohtsubo, H., and Aoh, K. (1979). "Stability and post-critical growth of a system of cooling and shrinkage cracks." Int. J. Fracture, $15,443-456$.

Bažant Z. P., and Pfeiffer, P. A. (1987). "Determination of fracture energy from size effect and brittleness number." ACI Mat. J., 84(6), 463-480.

Hillerborg, A. (1985). "Results of three comparative test series for determining the fracture energy $G_{f}$ of concrete." Mat. and Struct. $18(107), 407-413$.

Hillerborg, A., Modéer, M., and Petersson, P. E. (1976). "Analysis of crack formation and crack growth in concrete by means of fracture mechanics and finite elements." Cement and Concrete Res., 6, 773782.

Jeng, Y. S., and Shah, S. P. (1985). "A two parameter fracture model for concrete." J. Engrg. Mech., ASCE, 111(4), 1227-1241.

Li, Y. N., and Bažant, Z. P. (1994). "Penetration fracture of floating iceplate: 2D analysis and size effect." J. Engrg. Mech., ASCE, 120(7), $1481-1498$.

Li, Y. N., Hong, A. P., and Bažant, Z. P. (1995). "Initiation of parallel cracks on the surface of an elastic half-plane." Int. J. Fracture, 69 $357-369$.

Okamura, H., Watanabe, K., and Takano, T. (1972). "Applications of the compliance concept in fracture mechanics." Proc., 6th Nat. Symp. on Fracture Mech., Progress in Flaw Growth and Fracture Toughness Testing, Spec. Tech. Publ. No. 536, Am. Soc. of Mech. Engrs. (ASME) New York, N.Y., 423-439.

Planas, J., and Elices, M. (1991). "Nonlinear fracture of cohesive materials." Int. J. Fracture, 51, 139-157.

Rice, J. R., and Levy, N. (1972). "The part-through surface crack in an elastic plate." J. Appl. Mech., 39, $185-194$.

Ruth, B. E., Bloy, L. A. K., and Avital, A. A. (1982). "Prediction of pavement cracking at low temperature." Asphalt Paving Technol. (Proc., Assoc. of Asphalt Paving Technologists, Tech. Sessions), Vol. $51,53-103$.

Tada, H., Paris, P. C., and Irwin, G. R. (1985). Stress analysis of cracks handbook. Del Research Corp., Hellertown, Pa.

where 